\title{
1 Virtual Reality in Biology: Can We Become Virtual Naturalists?
}

2 Authors list: Juliano Morimoto ${ }^{1,2, *}$, Fleur Ponton $^{1}$

3

4 Author's affiliations:

51 - Department of Biological Sciences, Macquarie University, NSW 2109, North Ryde,

6 Australia

72 - School of Biological Sciences, University of Aberdeen, Zoology Building, Tillydrone

8 Ave, Aberdeen AB24 2TZ, United Kingdom

$9 \quad *$ To whom correspondence should be addressed:

10 Juliano Morimoto

11 School of Biological Sciences, University of Aberdeen, Zoology Building, Tillydrone Ave,

12 Aberdeen AB24 2TZ, United Kingdom, e-mail: juliano.morimoto@gmail.com

14 Abstract

15 Technological advances made Virtual and Mixed Reality (VMR) accessible at our fingertips.

16 However, only recently VMR has been explored for the teaching of biology. Here, we

17 highlight how VMR applications can be useful in biology education, discuss about caveats

18 related to VMR use that can interfere with learning, and look into the future of VMR

19 applications in the field. We then propose that the combination of VMR with Machine

20 Learning and Artificial Intelligence can provide unprecedented ways to visualise how species

21 evolve in self-sustained immersive virtual worlds, thereby transforming VMR from an

22 educational tool to the centre of biological interest.

23

24 Keywords : evolutionary biology / education / immersive reality 
25

26

27

\section{Introduction}

With increasing computational power, technologies that were costly or impossible to implement in the past have now become accessible in laptops and mobile phones $[1,2]$. These technologies are now revolutionising the ways we interact with the world, how we learn, and how we teach [3]. Virtual and Mixed Reality (VMR) is one of these technologies. Throughout the text, we use the term VMR to include all virtual types of applications, from Augmented Reality (AR), Mixed Reality (MR) through to Virtual Reality in its strict sense (see Box 1).

VMR can be defined as an alternate world filled with computer-generated entities that interact with human sensory and motor systems to cause a sense of 'presence' or 'immersion' in the subject [4]. Despite its highly technological nature, VMR has been around for decades, and it thought to have its origin when, in the 1960s, Morton Heilig created one of the first immersive multi-sensory simulator that included stimuli such as sound, scent, wind and vibration (called 'Sensorama') [5,6]. Ever since, VR technology has advanced significantly to the point that today there exists many platforms for creating as well as experiencing VMR applications [e.g., [7-9]].

In the last decade, the use of VMR in teaching and learning has increased dramatically, and spans across a variety of subjects [10-13], including biology [14,15]. A meta-analysis of sixteen studies has shown that VMR surgical simulators decrease the time to complete surgical procedures, suggesting a more efficient surgical skill acquisition [16]. Furthermore, VMR improves the learning of tasks that require spatial and visual memory, observation, as well as control of emotional responses in stressful conditions [17]. Importantly, autistic children have been described as having positive engagement with VMR applications in educational settings [18], suggesting that VMR can be used in a wide range of contexts and function as an inclusive tool for the education of students with special needs. Therefore, VMR has the 
50 potential to become an important educational tool in our century [10]. But, what is the true 51 power of VMR in teaching and learning in biology? What can future developments in VMR 52 teach us about nature?

53

54 Here, we highlight the power and applicability of VMR by providing an overview of how

55 VMR applications are changing teaching and learning in biology. Next, we discuss the potential caveats associated with VMR applications and discuss how VMR use can hinder

57 (rather than help) teaching and learning. Lastly, we look into the future of VMR technology and discuss the directions in which future VMR developments can teach us about important principles of nature, in which VMR can act as an independent, self-sustained virtual experimental world. Having said that, we hope that this paper will help guide future developments in VMR applied to biology in a constructive manner.

\section{VMR uses in Biology education}

VMR has been used in both secondary and tertiary education biology courses [13,19-21]. A number of VMR applications attempt to reproduce the laboratory environment to students with otherwise no access to laboratory facilities, with demonstrated benefits over traditional lectures [see e.g., Labster [22]]. Other VMR applications were designed to give the students an immersive experience of more specific biological processes such as the cell structure [23], spatial orientation [24], and vision formation in animals [25]. Students report higher engagement and learning outcomes with immersive experiences offered by VMR applications, which is encouraging for the use of VMR in biology education $[11,13,14,26,27]$ (Fig 1). For example, [24] designed an immersive interactive VMR platform for visualisation and teaching of conformation and geometry of protein crystallographic structures, whereby the test group was able to identify characteristics and regions in the samples that were 
75 obfuscated in non-immersive programs [24]. Thus, innovative curricula that harness the power

76

77

78

79

80

81

82

83

84

85

86

87

90

91

92

93

94

95

96

97

98

99

of new technologies can provide significant benefits to the teaching and learning of biology

[28,29].

VMR applications could help learning and teaching of ecology by simulating field expeditions in which students have to identify plants and/or animals in virtual reality, as in non-immersive virtual field trips developed previously [e.g., [30,31]]. Students have in fact reported that non-immersive virtual field trips provide a useful complement to the real field trip and could be a powerful tool to prepare and revise real field trips (Spicer and Stratford 2001). This could also complement units of taxonomy of plants and animals as well as provide virtual field experience to the student prior to the real task, thereby amalgamating students' learning experience. In VMR, immersive scenarios could include representative environments from different ecosystems (e.g., Amazon rainforest, tundra, desert) in which the aim is to identify the greater number of plant species as well as the morphological traits that are shared amongst species.

It is important to mention that virtual systems have been developed to explore all aspects of biology education. For instance, pervious digital material has been designed for teaching and learning of astrobiology [for instance in the Habitable Worlds platform [15]], although not yet in the fully immersive platform of VMR. Habitable Worlds allows students to experience a inquiry-driven learning environment designed to enhance students' learning outcomes on science through observation and modelling of virtual systems [15]. The results are promising as more than $70 \%$ of students had grades average or higher, and student engagement significantly increased compared to benchmark [15]. As such, Habitable Worlds provides some guidelines for the design of digital platforms that could be transferable to VMR 
100 systems, including automated feedback tailored to the students' needs and student-educator

101

102

103

104

105

106

107

108

109

110

111

112

113

114

115

116

117

118

119

120

121

122

123

124 interactions (both in real-time and in forums) [15]. It will be interesting for future developments of Habitable Worlds to expand the educational content from astrobiology to other subjects within biology, as well as to include VMR experience and compare the performance of students with traditional versus immersive platforms.

\section{The potential misuses of VMR}

As for any new technology, we are still discovering the limitations of VMR applications as educational tools. VMR applications are attractive because they contain a wide variety of sensory stimuli that give the participant a sense of immersion (presence). However, too many stimuli - such as colours, shapes, characters, movement - can distract the participant and have detrimental effects on learning, a phenomenon that has been acknowledged in the literature and commonly referred to as cognitive overload [32]. A recent study has shown that university students learned less and experienced higher cognitive overload when they experienced a science lab in a fully-mounted VMR headset as oppose to the VMR scenes played on 2D displays, in spite of higher feeling of presence (i.e., immersion) in the VMR scene as opposed to the 2D screen display [27]. This suggests that, in some cases, the very same attributes that make VMR attractive can make VMR applications ineffective. Other negative effects of VMR applications are motion sickness and dizziness caused by the immersive experience [33-35], which can preclude appropriate understanding of the material. Given the negative effects of VMR, guidelines are urgently needed to minimise VMR misuses. Recent literature provides comprehensive lists of fundamental characteristics of 3D virtual environments and general features that can be adjusted to increase students' engagement and learning in virtual systems [see e.g., [36-38]]. Here, our point is to reiterate the importance of careful design and testing of new VMR applications prior to 
125 implementation in the classroom in order to mitigate cognitive overload and/or motion 126 sickness, which could significantly hamper VMR's educational potential [36]. Research is

127 only beginning to uncover the positive and negative aspects of VMR applications; future 128 studies will provide more detailed evidence-based guidelines to build effective VMR 129 applications that maximise educational potential while minimising negative effects of VMR 130 misuse [39,40].

The future: Can VMR teach us Biology?

133 The use of VMR technology in teaching and learning will very certainly be part of the future

134 of education across all disciplines, and the formulation of evidence-based guidelines for the creation of VMR educational material is urgently needed. While we can teach and learn

136 Biology using VMR applications, a key question is 'can VMR teach us anything about

137 Biology?' We believe - as described below - that the answer is 'Yes'. It is important to 138 clarify that we are not criticising previous work in the field but instead aimed at 139 conceptualising a new way of harnessing the power of new technologies such as VMR to 140 biology teaching and learning.

\section{Bio-inspired systems and the rise of artificial evolution}

143 The parallels between natural and artificial evolutionary systems have long been recognised

144 and explored. While few artificial life systems exist [41-43], perhaps the most famous example

145 comes from the work of Thomas Ray and the 'Tierra' system [44]. The Tierra system

146 simulates artificial life in self-replicating, evolving entities (aka 'algorithms') confined within

147 virtual computer spaces, whereby the entities can be considered as uni- or multi-cellular

148 entities that experience errors in replication analogous to mutations in biological reproduction

149 [44-46]. Instead of solar energy and natural resources as in biological systems, artificial 
150 entities compete for central processing unit (CPU) and memory space [analogous to energy

151 and spatial resource, respectively, as described in [44]]. As a result, artificial Tierra entities

152 become progressively more adapted to exploit one another in order to gain advantage over

153 the use of CPU and memory [44,47]. The outcome of this self-sustained virtual evolutionary

154 world is remarkable given that the system evolves differences in entity sizes, ecological

155 specialisation (e.g., parasites) and population dynamics processes (e.g., extinction) [44,46-48].

156 This provides an unprecedented study case to compare and understand how different shapes

157 and forms emerge through evolutionary processes. However, visualisation of evolution in the

158 Tierra system is not straightforward and largely inaccessible to a broader audience due to the

159 highly technical language underlying the system. This poses a significant barrier to biologists

160 with limited computational expertise and it is, to some extent, visually unappealing for

161 students of biological sciences and related disciplines. Consequently, it is difficult (though

162 not impossible) to use artificial model systems such as Tierra as an effective educational tool

163 in the classroom while keeping the attention span and interest of students.

165 Can VMR and Artificial Intelligence (AI) revolutionise artificial evolutionary systems?

166 As discussed above, VMR is a powerful and appealing educational technology to teach

167 biology. This is because students and educators respond rationally as well as emotionally to

168 the educational material in the immersive experience, which can accentuate learning

$169[21,22,31,49,50]$. Thus, VMR can be an appropriate way to overcome accessibility problems of

170 artificially evolving systems while increasing visual appealing to specialists and general

171 audience.

172

173 The technological advances that allowed VMR to become an accessible tool has also allowed

174 for powerful statistical models of Machine Learning and Artificial Intelligence (AI) to 
175 mushroom. Machine Learning are algorithms that process and learn with huge amounts of

176 data in order to perform a task without necessarily being explicitly programmed to do so [51].

177 AI attempts to simulate human intelligence in machine systems; this includes machine

178 learning but also (bio-inspired) robotics, ethics and philosophy associated with AI

179 development [52]. Importantly, AI advances have recently demonstrated that machines can

180 learn from data beyond human capabilities [53,54]. Furthermore, a new area on the interface

181 between VMR and AI aims to integrate AI to entities in VMR [55-57]. As a result, a key

182 question emerges: can we combine Machine Learning and AI with VMR to create a self-

183 sustained evolving virtual world (a 'BioVR')? If so, why should we combine VMR with AI?

184 The answer to the first question is, in our opinion, a sounding 'yes'. We strongly believe that

185 future technological advances have the potential to create an immersive virtual world that

186 reproduces the forces of evolution, which can allow us to visualise and measure how species

187 have evolved, how ecosystems are formed, how species adapt to their environment, how we

188 can anticipate effects of adverse climatic conditions across ecosystems in our changing

189 world. In a sense, we could become 'virtual naturalists'. The learning benefits are

190 unprecedented given that students can experience inaccessible and inhospitable

191 environments, observe evolution, adaptation, trophic interaction, parasitism and many more

192 biological processes without stepping outside the classroom. Furthermore, the freedom given

193 to the students within these BioVRs forms the perfect ground for inquiry-based learning,

194 where the students will observe and explore the environment, measuring and experiencing the

195 virtual environment to inquiry about the underlying virtual biological phenomena [15]. The

196 BioVR could then eliminate the need for complex computational expertise (at least from the

197 users' point of view) and provide a fully immersive, artificial world upon which entities

198 evolve following basic principles of biological evolution in our and other planets, while

199 students can explore the environment and learn from their own virtual experience. 


\section{BioVR concept}

202 We discuss here a series of possible steps upon which BioVR as we envisaged could be 203 brought to (artificial) life, or at least inspire specialists in the field to further develop the ideas 204 into a prototype of the concept. The steps are:

1. Simulate an artificial 'planet' whereby entities will interact, compete, and evolve. In this artificial planet, the 'biotic' rules are established, such as the basic environmental conditions (e.g., gravity) and resource distribution (e.g., marine vs terrestrial landscapes) [similar in concept to the 'soup' in Tierra [44] and the concept of virtual environments in [55]].

2. Design the ancestor entity, defining the rules of reproduction, mutation, and ecological interactions with the resources in the planet. The ancestor entity is the 'building block' for artificial life to evolve in BioVR and without it, the system does not have the evolving entity. The ancestor entity is equivalent to the ancestor species which gave origin to life on Earth, and is a common feature of artificial life systems [e.g., [46]]. In other words, without the ancestor entity to evolve, the system would resemble an immersive version of Google Earth [58].

3. Gather a large empirical dataset of environment-traits-species interactions as a basic starting-point for determining how different species evolve in different ecosystems (e.g., evolutionary convergences, divergences, character displacement) - we could call this 'rules of evolution'. One way in which evolutionary rules could be extracted from this dataset is using, for example, supervised learning models (see Box 2) to extract general rules as to how species evolve (morphologically and behaviourally) across different environments, commonality between functional traits across species in the same environments, as well as the number, distribution, and behaviour of 
different species within the same environment. Of course, this is optional as we may

226

227

228

229

230

231

232

233

234

235

236

237

238

239

240

241

242

243

244 want to allow the system free to create its own evolutionary rules along time (iterations). Nonetheless, we believe that, if feasible, a good first proof-of-concept prototype should be based on empirical data. Once these rules of evolution are estimated (or guessed), they are applied to the ancestral entity which is allowed to evolve.

4. Ideally, BioVRs are self-sustained, and thus it would be interesting to have the changes and adaptations in one time point fed-back into the system for the next time point. For example, imagine that a species evolves a remarkable adaptation to convert virtual resource A into B. This transformation should be fed-back into the system so as to allow new evolutionary rules, perhaps favouring other species to adapt and utilise virtual resource B (which is being produced) instead of virtual resource A. Note that, over time, the ancestral entity will then evolve and adapt to different virtual resources and environments, thereby simulating evolution in a fully immersive system (Fig 2).

5. Given this self-sustained cycle of interaction between entities and the environments, and the iterative system that modulates virtual evolutionary rules, BioVR can become an artificial ecosystem, fully accessible for exploration through VMR in inquiry-based learning quests. This allows students and researchers to experience and study evolution in this immersive environment, comparing the outcomes of evolutionary forces within different environments within a BioVR and across BioVRs with different setups. Furthermore, since data visualisation is key for understanding biological processes [e.g., [59]] and is an essential component of affective learning [49,50], the use of VR to create BioVR worlds will allow VR to transcend the status of an educational tool that helps learning and teaching in Biology to become the main 
250

251

252

253

254

255

256

257

258

259

260

261

262

263

264

265

266

267

268

269

270

271

272

273

274

technology for experiencing and learning about virtual biological phenomena. It is important to mention that BioVRs, have the potential to suffer from the same VMR misuses discussed above (e.g., cognitive overload) because the student and/or researchers can experience a highly immersive, dynamic and stimuli-rich virtual environment. One way that this could be minimised is by limiting the number of potential stimuli available at a given time; for instance, limit drastic changes in colour and texture of the scene simultaneously. Whether cognitive overload will affect BioVRs functionality remains contingent upon trials of first prototypes of the BioVR concept.

While the idea of BioVRs may seem allusive, attempts to merge the fields of VR, artificial life, and AI have been around for decades [55], with more recent efforts emerging from the astonishing 'boost' in computer power of our generation [60,61]. To our knowledge, concepts similar to the one of BioVR, as presented in this paper, have never been tested and thus remain subject of future developments in the field of statistics and computational biology.

Nonetheless, we are aware that virtual environments, AI and semi-autonomous VR agents have been developed for other purposes such as direct or assist users into tasks [see e.g., [55]], and we are positive that future endeavours in this field will allow BioVRs to be available as an educational tool in biology.

\section{Conclusion}

VMR can be a powerful ally in biology education. The use of VMR has provided promising results for consolidating learning across secondary and tertiary biology education. With increasing technology, the combination of VMR with Machine Learning and AI has the potential to create a self-sustained evolving virtual world (BioVR) that allow us to uniquely explore how life as we know evolves and responds to extreme climatic conditions. 


\section{Conflict of interest}

276 The authors have no conflict of interests to declare.

\section{References}

278 1. Kish, L. Moore's law and the energy requirement of computing versus performance. IEE Proceedings Circuits, Devices and Systems 2004, 151, 190-194.

280 2. Waldrop, M.M. The chips are down for Moore's law. Nature News 2016, 530, 144.

281 3. Veletsianos, G. Emerging technologies in distance education; Athabasca University

4. Yoh, M.-S. The reality of virtual reality. In Proceedings of Proceedings of the Seventh International Conference on Virtual Systems and Multimedia, 2001. ; pp. 666-674.

5. Heilig, M. Sensorama simulator, US patent no. 3050870. August: 1962.

6. Mazuryk, T.; Gervautz, M. Virtual reality: history, applications, technology and future. Citeseer 1996, 1.

7. Ledermann, F.; Schmalstieg, D. APRIL: a high-level framework for creating augmented reality presentations. In Proceedings of Virtual Reality, 2005. Proceedings. VR 2005. IEEE; pp. 187-194.

8. Jerald, J.; Giokaris, P.; Woodall, D.; Hartbolt, A.; Chandak, A.; Kuntz, S. Developing virtual reality applications with Unity. In Proceedings of Virtual Reality (VR), 2014 iEEE; pp. 1-3.

9. Wexelblat, A. Virtual reality: applications and explorations; Academic Press: Cambridge, MA, 2014.

10. Hoffman, H.; Vu, D. Virtual reality: teaching tool of the twenty-first century? Academic medicine: journal of the Association of American Medical Colleges 1997, 72, 1076-1081.

11. Mikropoulos, T.A.; Natsis, A. Educational virtual environments: A ten-year review of empirical research (1999-2009). Computers \& Education 2011, 56, 769-780.

12. Davies, A.G.; Crohn, N.J.; Treadgold, L.A. Can virtual reality really be used within the lecture theatre? BMJ Simulation and Technology Enhanced Learning 2018, bmjstel2017-000295.

13. Makransky, G.; Thisgaard, M.W.; Gadegaard, H. Virtual simulations as preparation for lab exercises: Assessing learning of key laboratory skills in microbiology and improvement of essential non-cognitive skills. PLOS ONE 2016, 11, e0155895.

14. Shim, K.-C.; Park, J.-S.; Kim, H.-S.; Kim, J.-H.; Park, Y.-C.; Ryu, H.-I. Application of virtual reality technology in biology education. Journal of Biological Education 2003, 37, 71-74.

15. Horodyskyj, L.B.; Mead, C.; Belinson, Z.; Buxner, S.; Semken, S.; Anbar, A.D. Habitable Worlds: Delivering on the promises of online education. Astrobiology 2018, 18, 8699.

16. Haque, S.; Srinivasan, S. A meta-analysis of the training effectiveness of virtual reality surgical simulators. IEEE Trans. Inf. Technol. Biomed. 2006, 10, 51-58.

17. Jensen, L.; Konradsen, F. A review of the use of virtual reality head-mounted displays in education and training. Education and Information Technologies 2018, 23, 15151529. 
18. Strickland, D.; Marcus, L.M.; Mesibov, G.B.; Hogan, K. Brief report: Two case studies using virtual reality as a learning tool for autistic children. J. Autism Dev. Disord. 1996, 26, 651-659.

19. Poland, R.; Baggott la Velle, L.; Nichol, J. The Virtual Field Station (VFS): using a virtual reality environment for ecological fieldwork in A-Level biological studiesCase Study 3. Br. J. Educat. Tech. 2003, 34, 215-231.

20. De Jong, T.; Linn, M.C.; Zacharia, Z.C. Physical and virtual laboratories in science and engineering education. Science 2013, 340, 305-308.

21. Thisgaard, M.; Makransky, G. Virtual learning simulations in high school: Effects on cognitive and non-cognitive outcomes and implications on the development of STEM academic and career choice. Frontiers in psychology 2017, 8, 805.

22. Bonde, M.T.; Makransky, G.; Wandall, J.; Larsen, M.V.; Morsing, M.; Jarmer, H.; Sommer, M.O. Improving biotech education through gamified laboratory simulations. Nat. Biotechnol. 2014, 32, 694.

23. McClean, P.E.; Slator, B.M.; White, A.R. The virtual cell: An interactive, virtual environment for cell biology. In Proceedings of EdMedia: World Conference on Educational Media and Technology; pp. 1442-1443.

24. Moritz, E.; Meyer, J. Interactive 3D protein structure visualization using virtual reality. In Proceedings of Proceedings of the Fourth IEEE Symposium on Bioinformatics and Bioengineering, 2004. BIBE 2004. ; pp. 503-507.

25. Dominy, N.; Gochman, S. Tarsier Goggles: a virtual reality tool for experiencing the optics of a dark-adapted primate visual system. DArmouth Digital Commons 2018.

26. Mikropoulos, T.A.; Katsikis, A.; Nikolou, E.; Tsakalis, P. Virtual environments in biology teaching. Journal of Biological Education 2003, 37, 176-181.

27. Makransky, G.; Terkildsen, T.S.; Mayer, R.E. Adding immersive virtual reality to a science lab simulation causes more presence but less learning. Learning and Instruction 2017.

28. Eastwood, J.L.; Sadler, T.D. Teachers' implementation of a game-based biotechnology curriculum. Computers \& Education 2013, 66, 11-24.

29. Sadler, T.D.; Romine, W.L.; Menon, D.; Ferdig, R.E.; Annetta, L. Learning Biology through innovative curricula: a comparison of game-and nongame-based approaches. Science Education 2015, 99, 696-720.

30. Spicer, J.I.; Stratford, J. Student perceptions of a virtual field trip to replace a real field trip. J. Comput. Assisted Learn. 2001, 17, 345-354.

31. Minocha, S.; Tudor, A.-D.; Tilling, S. Affordances of mobile virtual reality and their role in learning and teaching. In Proceedings of 31st British Human Computer Interaction Conference, University of Sunderland's St. Peter's Campus, UK.

32. Whitelock, D.; Romano, D.; Jelfs, A.; Brna, P. Perfect presence: What does this mean for the design of virtual learning environments? Education and Information Technologies 2000, 5, 277-289.

33. McCauley, M.E.; Sharkey, T.J. Cybersickness: Perception of self-motion in virtual environments. Presence: Teleoperators \& Virtual Environments 1992, 1, 311-318.

34. Regan, E.; Price, K. The frequency of occurrence and severity of side-effects of immersion virtual reality. Aviat. Space Environ. Med. 1994.

35. Ohyama, S.; Nishiike, S.; Watanabe, H.; Matsuoka, K.; Akizuki, H.; Takeda, N.; Harada, T. Autonomic responses during motion sickness induced by virtual reality. Auris. Nasus. Larynx 2007, 34, 303-306. 
402

403

404

405

406

407

408

409

410

36. Dalgarno, B.; Lee, M.J. What are the learning affordances of 3-D virtual environments? Br. J. Educat. Tech. 2010, 41, 10-32.

37. Lindgren, R.; Tscholl, M.; Wang, S.; Johnson, E. Enhancing learning and engagement through embodied interaction within a mixed reality simulation. Computers \& Education 2016, 95, 174-187.

38. O'Connor, E.A.; Domingo, J. A practical guide, with theoretical underpinnings, for creating effective virtual reality learning environments. Journal of Educational Technology Systems 2017, 45, 343-364.

39. Dunleavy, M.; Dede, C.; Mitchell, R. Affordances and limitations of immersive participatory augmented reality simulations for teaching and learning. Journal of science Education and Technology 2009, 18, 7-22.

40. Akçayır, M.; Akçayır, G. Advantages and challenges associated with augmented reality for education: A systematic review of the literature. Educational Research Review 2017, 20, 1-11.

41. Langton, C.G. Studying artificial life with cellular automata. Physica D: Nonlinear Phenomena 1986, 22, 120-149.

42. Langton, C.G. Artificial life: An overview; Mit Press: Cambridge, MA, 1997.

43. Lehman, J.; Clune, J.; Misevic, D.; Adami, C.; Altenberg, L.; Beaulieu, J.; Bentley, P.J.; Bernard, S.; Beslon, G.; Bryson, D.M. The surprising creativity of digital evolution: A collection of anecdotes from the evolutionary computation and artificial life research communities. arXiv preprint arXiv:1803.03453 2018.

44. Ray, T.S. Evolution, ecology and optimization of digital organisms. In Scientific Excellence in Supercomputing: The IBM 1990 Contest Prize Papers, Billingsley, D., Brown, Ed. Citeseer: Athens, GA, 1992; Vol. 1.

45. Thearling, K.; Ray, T.S. Evolving multi-cellular artificial life. In Artificial Life IV, Maes, B.a., Ed. MIT Press: Cambridge, MA, 1994; pp. 283-288.

46. Ray, T.S. Evolution, complexity, entropy and artificial reality. Physica D: Nonlinear Phenomena 1994, 75, 239-263.

47. Ray, T.S.; Xu, C. Measures of evolvability in tierra. Artif. Life Robot. 2001, 5, 211-214.

48. Shao, J.; Ray, T.S. Maintenance of species diversity by predation in the Tierra system. In Proceedings of Proceedings of the Alife XII Conference, Odense, Denmark; pp. 533-540.

49. Harley, J.M.; Poitras, E.G.; Jarrell, A.; Duffy, M.C.; Lajoie, S.P. Comparing virtual and location-based augmented reality mobile learning: emotions and learning outcomes. Educational Technology Research and Development 2016, 64, 359-388.

50. Riva, G.; Mantovani, F.; Capideville, C.S.; Preziosa, A.; Morganti, F.; Villani, D.; Gaggioli, A.; Botella, C.; Alcañiz, M. Affective interactions using virtual reality: the link between presence and emotions. CyberPsychol. Behav. 2007, 10, 45-56.

51. Bishop, C.M. Pattern recognition and machine learning; Springer-Verlag New York: 2006; Vol. 1, pp. 738.

52. Russell, S.J.; Norvig, P. Artificial intelligence: a modern approach; Pearson Education Limited: Upper Saddle River New Jersey, 2016.

53. Chouard, T. The Go Files: Al computer wraps up 4-1 victory against human champion. Nature News 2016.

54. Gibney, E. Google Al algorithm masters ancient game of Go. Nature News 2016, 529, 445. 
411

412

413

414

415

416

417

418

419

420

421

422

423

424

425

426

427

428

429

430

431

432

433

434

435

436

437

438

439

440

441

442

443

444

445
55. Luck, M.; Aylett, R. Applying artificial intelligence to virtual reality: Intelligent virtual environments. Applied Artificial Intelligence 2000, 14, 3-32.

56. Laukkanen, S.; Karanta, I.; Kotovirta, V.; Markkanen, J.; Rönkkö, J. Adding intelligence to virtual reality. In Proceedings of Proceedings of the 16th European Conference on Artificial Intelligence; pp. 1136-1141.

57. Augusto, J.C.; Callaghan, V.; Cook, D.; Kameas, A.; Satoh, I. Intelligent environments: a manifesto. Human-Centric Computing and Information Sciences 2013, 3, 12.

58. Gorelick, N.; Hancher, M.; Dixon, M.; Ilyushchenko, S.; Thau, D.; Moore, R. Google Earth Engine: Planetary-scale geospatial analysis for everyone. Remote Sens. Environ. 2017, 202, 18-27.

59. Karr, T.L.; Brady, R. Virtual biology in the CAVE. Trends Genet. 2000, 16, 231-232.

60. Petrović, V.M. Artificial Intelligence and Virtual Worlds-Toward Human-Level Al Agents. IEEE Access 2018, 6, 39976-39988.

61. Zhang, Z.; Cao, B.; Guo, J.; Weng, D.; Liu, Y.; Wang, Y. Inverse virtual reality: intelligence-driven mutually mirrored world. In Proceedings of 2018 IEEE Conference on Virtual Reality and 3D User Interfaces (VR); pp. 735-736.

62. Milgram, P.; Takemura, H.; Utsumi, A.; Kishino, F. Augmented reality: A class of displays on the reality-virtuality continuum. In Proceedings of Telemanipulator and Telepresence Technologies; pp. 282-293.

63. Serino, M.; Cordrey, K.; McLaughlin, L.; Milanaik, R.L. Pokémon Go and augmented virtual reality games: a cautionary commentary for parents and pediatricians. Curr. Opin. Pediatr. 2016, 28, 673-677.

64. Zsila, Á.; Orosz, G.; Bőthe, B.; Tóth-Király, I.; Király, O.; Griffiths, M.; Demetrovics, Z. An empirical study on the motivations underlying augmented reality games: The case of Pokémon Go during and after Pokémon fever. Personality and Individual Differences 2018, 133, 56-66.

65. Michalski, R.S.; Carbonell, J.G.; Mitchell, T.M. Machine learning: An artificial intelligence approach; Springer Science \& Business Media: Berlin, Germany, 2013.

66. Zhu, X.; Goldberg, A.B. Introduction to semi-supervised learning. Synthesis Lectures on Artificial Intelligence and Machine Learning 2009, 3, 1-130.

\section{Figure captions}




\begin{tabular}{|c|c|}
\hline \multicolumn{2}{|c|}{ Virtual Reality (VR) } \\
\hline & \multirow{2}{*}{$\begin{array}{c}\text { Machine Learning } \\
\text { Artificial Intelligence }\end{array}$} \\
\hline \multirow{2}{*}{$\begin{array}{l}\text { Teaching \& Learning } \\
\text { in Biology }\end{array}$} & \\
\hline & $\downarrow$ \\
\hline \multirow[b]{2}{*}{$\begin{array}{ll}\text { - } & \text { Positive learning } \\
& \text { outcomes } \\
\text { - } & \text { Higher student } \\
& \text { engagement } \\
\text { - } & \text { Preparation and } \\
& \text { Review of real field } \\
& \text { experiences } \\
\text { - } & \text { Virtual lab learning }\end{array}$} & BIOVR \\
\hline & $\begin{array}{l}\text { - } \begin{array}{l}\text { Self-sustained evolving virtual } \\
\text { world }\end{array} \\
\text { - } \quad \text { Increased understanding of } \\
\text { evolution } \\
\text { - } \quad \text { Simulation of evolution in extreme } \\
\text { environments (e.g., other planets) } \\
\text { - Powerful visualisation tool }\end{array}$ \\
\hline
\end{tabular}

447 Fig 1 - Schematic overview of the potential for VMR to impact Biology. On one hand,

448 VMR has increasingly been used for teaching of a variety of topics within Biology. As

449 technology advances, it may be possible to combine other cutting-edge technologies such as

450 Machine Learning and Artificial Intelligent to create a self-sustained evolving virtual world

451 (BioVR) that allows us to gain insights into biological processes.

452

Initial empirical rules of evolution

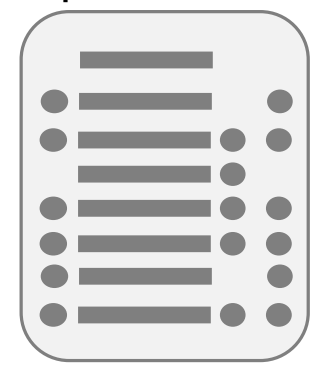

Environment-traitsspecies datasets
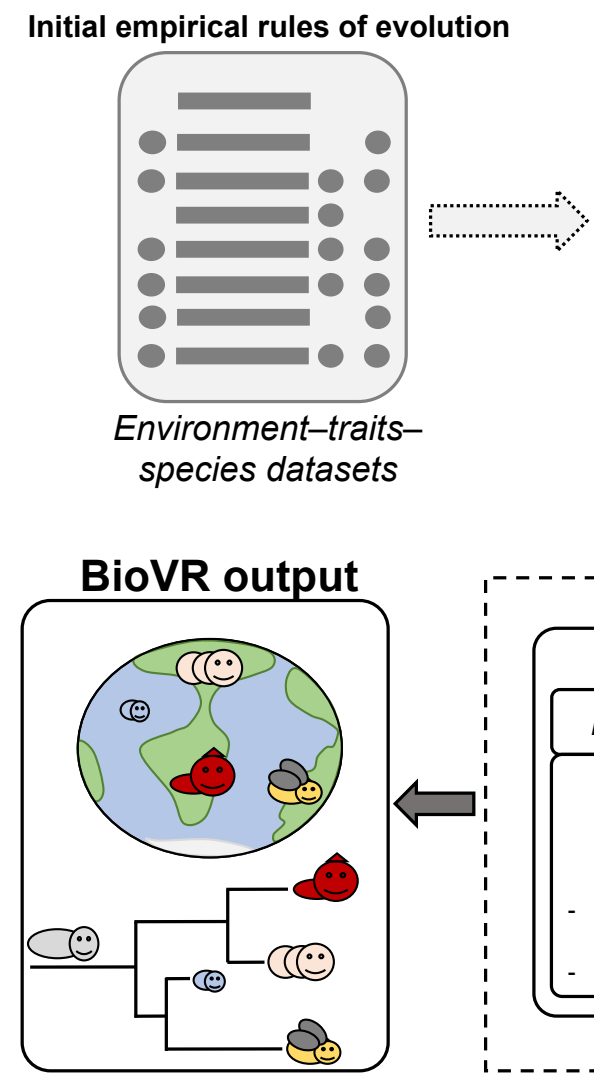

Evolving BioVR
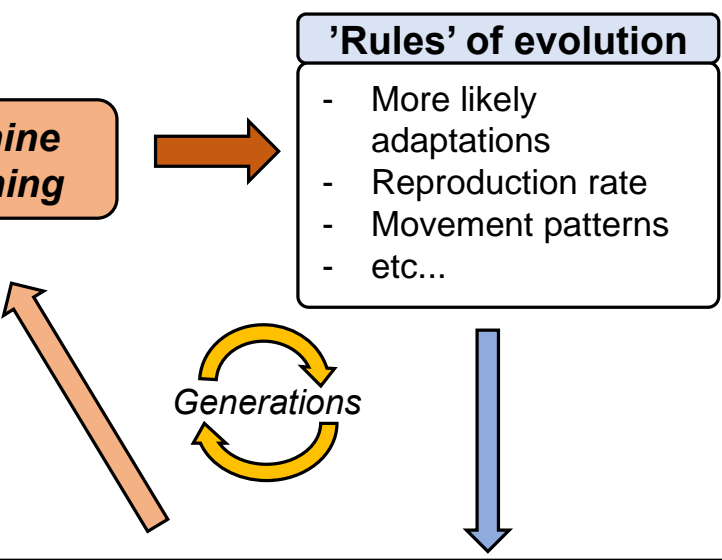

BioVR populated with Al entities

\begin{tabular}{|c|c|}
\hline BioVR initial conditions & Al entity \\
\hline $\begin{array}{l}\text { Initial physical and environmental } \\
\text { virtual conditions } \\
\text { Virtual ecosystems }\end{array}$ & $\begin{array}{ll}- & \text { Initial pre-defined life-history traits } \\
- & \text { Initial heritability of traits } \\
- & \text { Mutation rate } \\
- & \text { Rules of reproduction (two sexes?) }\end{array}$ \\
\hline
\end{tabular}


454 Fig 2 -Conceptual overview of the steps to build a BioVR. A supervised machine learning

455 algorithm is implemented to empirical environment-trait-species datasets in order to extract

456 the patterns (or 'rules') of evolution across environments. Meanwhile, the initial settings for

457 the BioVR world and the ancestral AI entity are also set. The settings include physical and

458 environmental conditions, as well as patterns of lifespan, movement, and reproduction of the

459 AI entity. Next, the 'rules of evolution' are incorporated into the BioVR and AI entity with

460 original settings, and the BioVR is allowed to evolve. Note that the evolution patterns in the

461 BioVR are then fed-back to the machine learning model, which is updated. This way, the

462 only input from empirical data is at the initial states, and BioVR are allowed to evolve

463 independently afterwards. As a result, we can measure and visualise species evolution as it

464 happens, in an immersive experience of the BioVR.

465

466

467 Boxes

468 Box 1 - The reality-virtuality continuum

469 In a highly influential paper, [62] proposed the reality-virtuality continuum (see Fig 3) to

470 classify VMR technology and applications. On one side of the spectrum is the real world

471 (reality) and, on the other side of the spectrum, the fully virtual world (virtuality) where

472 Virtual Reality (VR) in its strict sense resides. In between the extremes, stands Augmented

473 Reality (AR) - which relies mostly on real world elements but with the addition of virtual

474 entities; the best known (and controversial) example of AR has been Pokemon Go! [63,64]-

475 and Augmented Virtuality (AV) with the opposite of AR, that is, mostly virtual world but

476 with the addition of 'real' entities [62]. AR and AV are cases of Mixed Reality (MR), where

477 real and virtual elements are intertwined within the application (see Fig 3). For the purpose of 
478 this paper and for simplicity, we refer to AR, AV, and VR all as virtual and mixed reality

479 (VMR) applications because they all have some degree of virtuality.

480

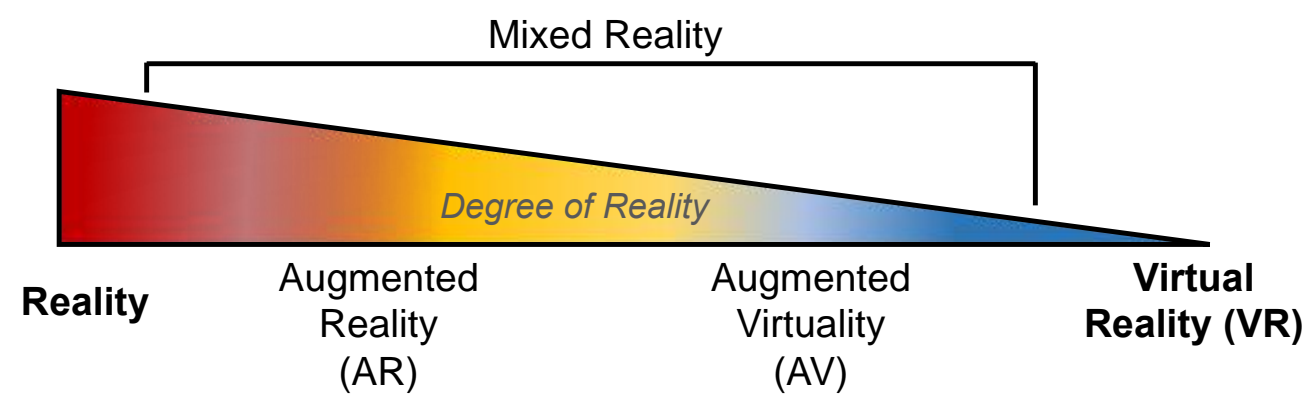

481 Fig 3 - The reality-virtuality continuum. AR - augmented reality; AV - augmented

482 virtuality; VR - virtual reality (based on Milgram et al., 1995).

483

\section{Box 2 - Supervised and unsupervised machine learning.}

485 Machine learning models can be broadly classified into supervised or unsupervised learning

486 algorithms, depending on the structure of the data [65] (Note: there are intermediate cases

487 called semi-supervised learning which we will not consider here, see e.g., [66] for details).

488 Unsupervised learning algorithms use data in which the outcome is not yet labelled or

489 identified, and therefore the algorithm cannot 'know' the outcomes in advance. The

490 algorithm then learns how to classify and predict the outcome from new observations based

491 on the inherent structure of the data at hand. An example of unsupervised learning is the

492 clustering of groups within a dataset (Fig 4a). Conversely, supervised learning algorithms

493 uses data in which the outcome is known, and the algorithm learns how to predict the

494 outcome of future observations based on what was learnt from the information and outcomes

495 obtained from previous data. An example of supervised learning is the classification (or

496 prediction, in the case of regression models) of a new observation between two categories

497 based on $n$ number of characteristics or variables (Fig 4b). 
a)

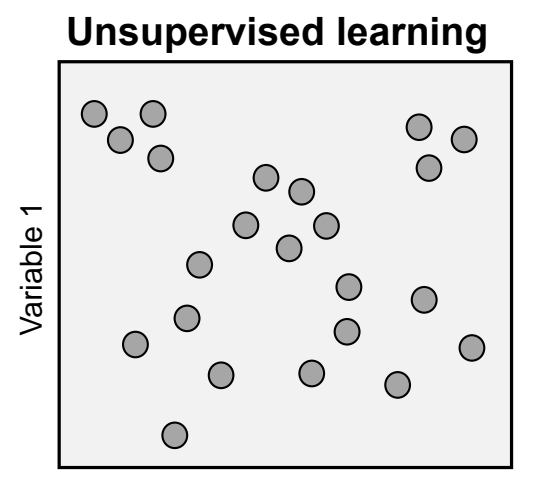

Variable 2

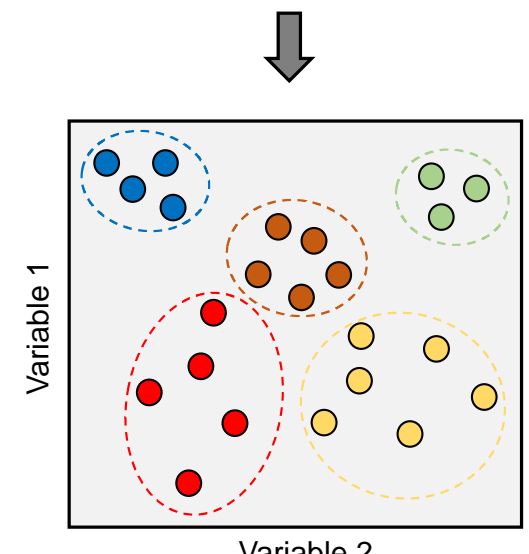

b) Supervised learning

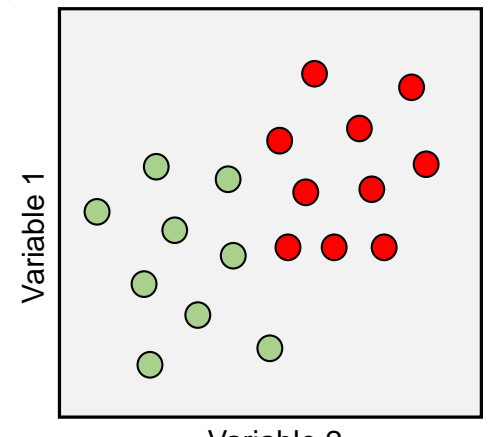

Variable 2

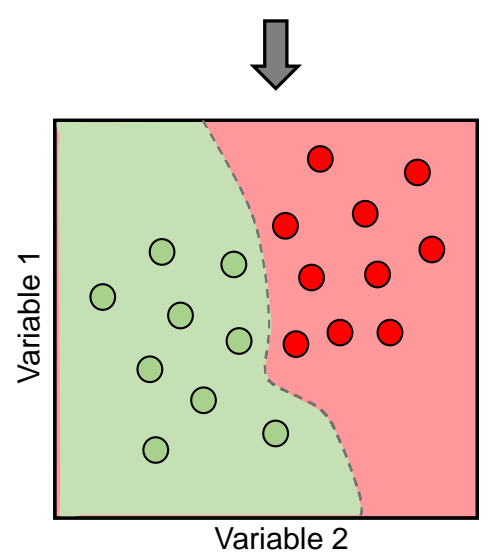

498

499 Fig 4-Supervised and unsupervised machine learning. a) Schematic representation of an

500 unsupervised learning model. Unlabelled data is used in unsupervised learning algorithms for

501 clustering. b) Schematic representation of a supervised learning model. Labelled data are used in supervised learning algorithms for classification.

\section{Biographical narrative}

505 Juliano Morimoto is a Research Fellow in the School of Biological Sciences at the

506 University of Aberdeen. He obtained his BSc in Biological Science (Brazil) during which he

507 was an undergraduate fellow at the Italian Synchrotron in Trieste. Dr Morimoto obtained his

508 DPhil (PhD) in Zoology at the University of Oxford, and completed two postdocs at the

509 University of Sydney and Macquarie University in Australia before his current appointment.

510 Dr Morimoto is coordinator and author of the upcoming book Poetic Geometry, funded by

511 the Brazilian National Research Council (CNPq), which is an initiative to disseminate 
512 scientific contents to high school children of low-income schools in Brazil. Dr Morimoto's

513 research is focused on behavioural ecology of invertebrates, particularly Diptera. He uses

514 Machine Learning and other statistical tools to model animal foraging behaviour and

515 decision-making in nutritional environments, and the life-history implications of these

516 choices to individuals and populations. Fleur Ponton is a Senior Lecturer in the Department

517 of Biological Sciences at Macquarie University. The primary goal of her research is to

518 describe and understand the network of interactions that defines the relationships between

519 nutrition, infection, and host fitness. The framework and methods originated from Dr

520 Ponton's research has opened a new avenue in the study of insect-symbiont interactions, and

521 have been applied across disciplines including nutritional sciences and ecology, animal

522 behaviour, as well as agricultural health. Dr Ponton is coordinates teaching units with more

523 than 450 students on the essential skills students should acquire to continue their studies in

524 Biology, and is fascinated in developing new tools to increase teaching effectiveness. 